\title{
Trombose venosa em boca: relatos de casos
}

\author{
Venous thrombosis in mouth: cases report \\ Trombose venosa en boca: relatos de casos \\ Rosana Mara Giordano de BARROS ${ }^{1}$ \\ Daniella Moraes ANTUNES ${ }^{1}$ \\ Kimberly Lampa GUSMÃO ${ }^{2}$ \\ Ângela Hassessian CARRILHO ${ }^{3}$ \\ Silvia Roberta Cieslak SANCHES ${ }^{4}$
}

${ }^{1}$ Professoras Doutoras em Patologia Bucal, Faculdade de Odontologia, Universidade Federal de Mato Grosso do Sul - Campo Grande (MS), Brasil

${ }^{2}$ Graduanda em Odontologia, Faculdade de Odontologia, Universidade Federal de Mato Grosso do Sul - Campo Grande (MS), Brasil

${ }^{3}$ Professora Mestre em Estomatologia, Faculdade de Odontologia, Universidade Federal de Mato Grosso do Sul - Campo Grande (MS), Brasil

${ }^{4}$ Responsável técnica pelo Laboratório de Patologia Bucal, Faculdade de Odontologia, Universidade Federal de Mato Grosso do Sul - Campo Grande (MS), Brasil

\section{Resumo}

Trombose é o processo patológico caracterizado pela solidificação do sangue dentro de vasos ou artérias e que raramente ocorre em cavidade oral. Geralmente assintomáticos, os trombos podem acometer qualquer local do sistema cardiovascular e sua ocorrência está associada a fatores de risco como a embolização. O presente artigo relata o caso atípico de dois pacientes diagnosticados com trombo venoso em boca, localizados em lábio superior esquerdo e em mucosa jugal inferior esquerda. Em ambos os casos, a conduta realizada foi biópsia excisional e após o resultado histopatológico, os pacientes foram encaminhados ao cardiologista, a fim de verificar a existência de outras lesões não visíveis ao exame físico. Devido ao difícil diagnóstico e baixa incidência em boca, a maioria dos cirurgiões dentistas não levam como principal hipótese diagnóstica o trombo venoso. Para tanto, quanto mais precocemente for realizado o diagnóstico, maior a possibilidade de aplicar medidas primordiais para controles adequados, evitar complicações e possíveis sequelas.

Descritores: Trombose; Lesões do Sistema Vascular; Boca.

\section{Abstract}

Thrombosis is the pathological process characterized by the solidification of blood within vessels or arteries and which rarely occurs in the oral cavity. Generally asymptomatic, thrombi may affect any site of the cardiovascular system and its occurrence is associated with risk factors such as embolization. This paper reports the atypical case of two patients diagnosed with venous thrombus in the mouth, located in the left upper lip and lower left jugal mucosa. In both cases, the procedure performed was excisional biopsy and after the histopathological result, the patients were referred to the cardiologist in order to verify the existence of other lesions not visible to the physical examination. Due to the difficult diagnosis and low incidence in the mouth, most dental surgeons do not take the venous thrombus as the main diagnostic hypothesis. For this, the earlier the diagnosis is made, the greater the possibility of applying primordial measures for adequate controls, avoiding complications and possible sequels.

Descriptors: Thombosis; Vascular System Injuries; Mouth.

\section{Resumen}

Trombosis es el proceso patológico caracterizado por la solidificación de la sangre dentro de vasos o arterias y que raramente ocurre en cavidad oral. Generalmente asintomáticos, los trombos pueden acometer cualquier sitio del sistema cardiovascular y su ocurrencia está asociada a factores de riesgo como la embolización. El presente artículo relata el caso atípico de dos pacientes diagnosticados con trombo venoso en boca, localizados en labio superior izquierdo y en mucosa yugo inferior izquierda. En ambos casos, la conducta realizada fue biopsia excisional y después del resultado histopatológico, los pacientes fueron encaminados al cardiólogo, a fin de verificar la existencia de otras lesiones no visibles al examen físico. Debido al difícil diagnóstico y baja incidencia en la boca, la mayoría de los cirujanos dentistas no llevan como principal hipótesis diagnóstica el trombo venoso. Para ello, cuanto más precozmente se realiza el diagnóstico, mayor es la posibilidad de aplicar medidas primordiales para controles adecuados, evitar complicaciones y posibles secuelas.

Descriptores: Trombosis; Lesiones del Sistema Vascular; Boca.

\section{INTRODUÇÃO}

As tromboses são doenças multifatoriais ${ }^{1}$, caracterizadas por obstruções completas ou parciais de um vaso ou artéria $^{2}$, devido a um processo patológico de solidificação do sangue quando há ativação anormal da coagulação sanguínea, desencadeada por lesões endoteliais, alterações no fluxo sanguíneo e/ou hipercoagulabilidade ${ }^{3}$.

O trombo venoso, na ausência de inflamação, desenvolve a flebotrombose. Contudo, na presença de reação inflamatória, ocorre a formação de tromboflebite ${ }^{4}$. Sua ocorrência geralmente está associada a um fator de risco para o desenvolvimento de trombose venosa profunda ${ }^{1-4}$. Em boca, os locais mais frequentes são, respectivamente, lábios, língua, mucosa jugal, mucosa alveolar, gengiva e assoalho bucal ${ }^{1}$.

São considerados fatores de risco para o desenvolvimento ou progresso do tromboembolismo venoso: traumas não cirúrgicos e cirúrgicos, idade superior a 40 anos, imobilização, alterações no fluxo sanguíneo, insuficiência cardíaca, infarto do miocárdio, paralisia de membros inferiores, obesidade, veias varicosas, uso de anticoncepcionais orais contendo estrógeno, gravidez, puerpério, doença pulmonar obstrutiva crônica, entre outros ${ }^{1}$.

Com complicações potencialmente fatais ${ }^{5}$, as manifestações clínicas variam de acordo com a gravidade do caso $^{6}$. A cavidade cardíaca e artéria aorta dificilmente são obstruídas pelos trombos, devido ao grande calibre e rápido fluxo sanguíneo da região. Todavia, a luz de artérias menores e veias podem ser completamente obstruídas ${ }^{7}$.

A proposição deste trabalho é descrever o processo de diagnóstico de trombos vasculares em boca, além de apresentar a importância de sua correta identificação para a adoção de medidas e controles adequados.

\section{CASOS CLÍNICOS}

- Caso 1

Paciente L.C.M., sexo masculino, 65 anos de idade, não fumante, raça branca, portador de prótese parcial removível superior, com lesão nodular unilateral em lábio superior lado esquerdo, $1,2 \mathrm{~cm}$ de 
diâmetro, firme à palpação, base séssil e de coloração vermelho-azulada (Figura 1). Durante a anamnese, o paciente relatou histórico médico de hipertensão arterial e uso contínuo de sua medicação. Devido às características clínicas, a suspeita diagnóstica inicial foi de hiperplasia ou adenoma pleomórfico.

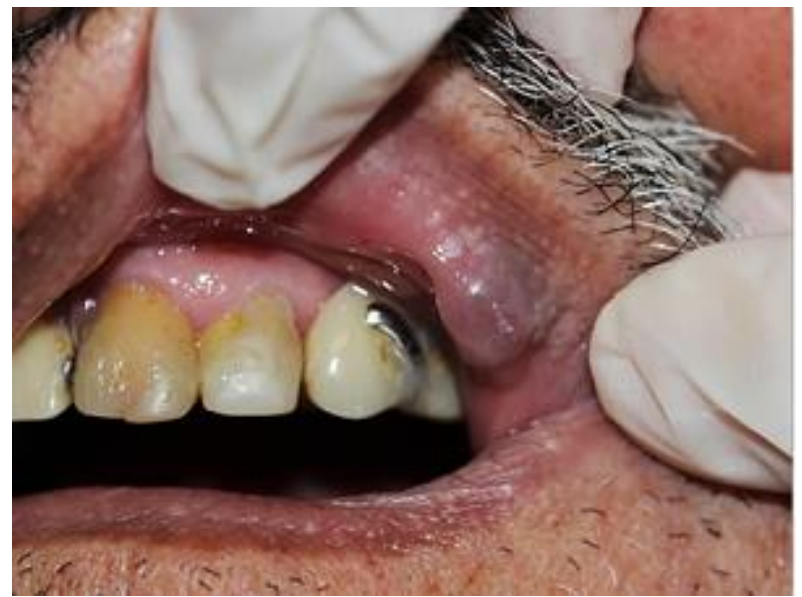

Figura 1: Características clínicas da lesão.

\section{- Caso 2}

Paciente do sexo masculino, 73 anos de idade, não fumante, pardo, com lesão nodular unilateral em mucosa jugal inferior lado esquerdo, 05 mm de diâmetro, elevada, arroxeada, assintomática, consistência flácida e com mobilidade à pressão (Figura 2). Durante a anamnese, o paciente relatou ter notado a lesão há cerca de um ano e que não utilizava nenhuma medicação. Devido às características clínicas, a suspeita diagnóstica inicial foi de lipoma, mucocele, trombo ou sialolitíase.

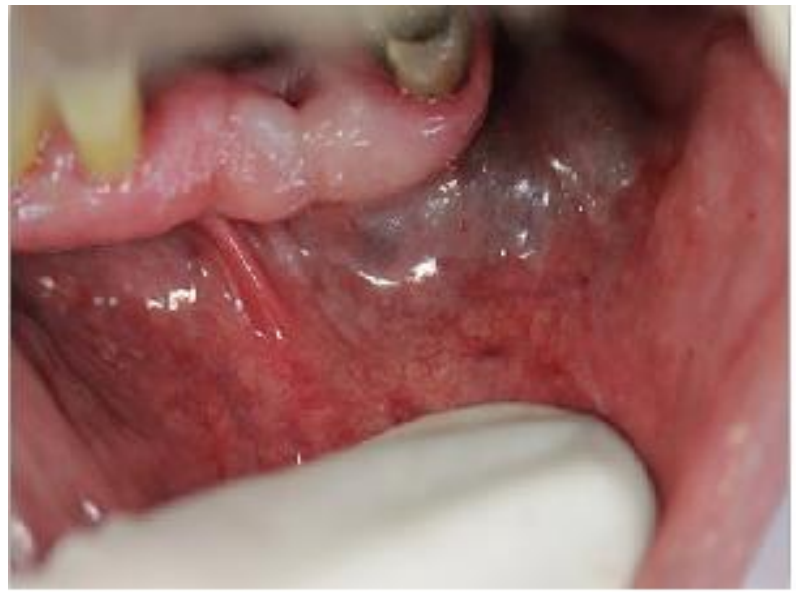

Figura 2: Características clínicas da lesão.

Os pacientes foram encaminhados ao projeto de Patologia Bucal da Faculdade de Odontologia da Universidade Federal de Mato Grosso do Sul e, em ambos os casos, a conduta realizada foi biópsia excisional. A histopatologia revelou, no tecido conjuntivo do fragmento avaliado, vasos venosos parcialmente obstruídos por massas de tecidos aderidas à parede vascular com centro abundantemente celular e periferia composta por hemácias e fibrina (Figuras 3 e 4, respectivamente). Os pacientes foram esclarecidos e encaminhados ao cardiologista para devida avaliação sistêmica. Até o momento, o pacientes encontram-se em proservação e nada mais foi observado, local e sistemicamente.

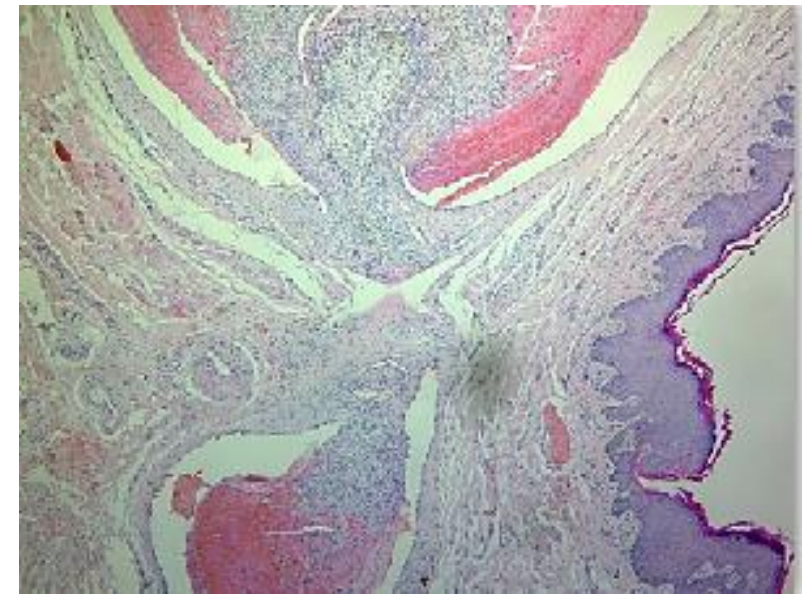

Figura 3: Histopatologia dos trombos vasculares. Aumento 40x. Técnica de HE.

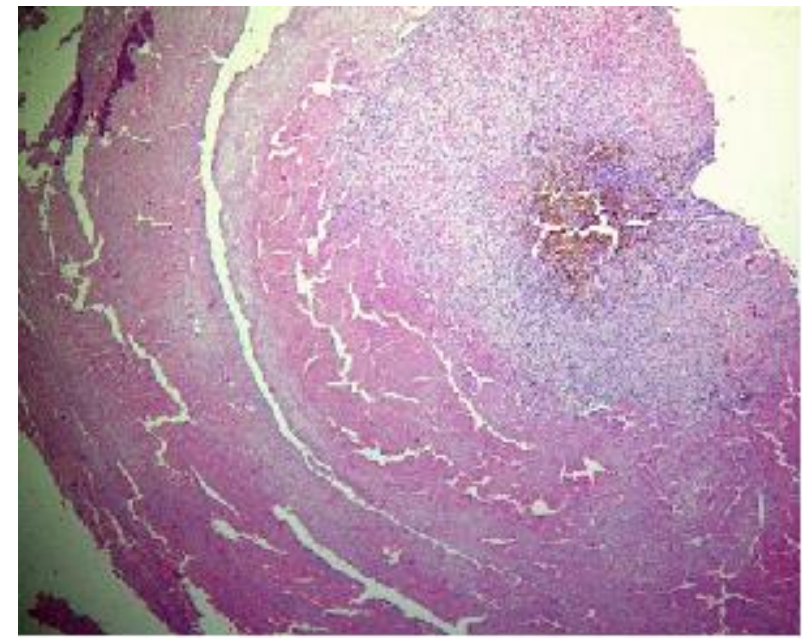

Figura 4: Histopatologia do trombo vascular. Aumento 40x. Técnica de HE.

\section{DISCUSSÃO}

A trombose pode ser fatal ${ }^{4}$, sobretudo quando ocorrem processos de embolização, que obstruem o fluxo sanguíneo a órgãos essenciais, como coração, pulmão ou cérebro. Em caso de trombose da artéria carótida interna ou de artérias cerebrais, AVC ou complicações neurológicas podem ser desencadeadas $^{8}$. Pela possibilidade do trombo ser transportado para qualquer local do sistema cardiovascular, o tratamento dependerá do tamanho e do lugar em que o êmbolo se instalou.

Lesões vasculares orais são geralmente assintomáticas $^{9}$, sua coloração pode variar do vermelho ao roxo, de acordo com a localização e a profundidade da invasão tecidual, bem como o grau de congestão vascular da área afetada ${ }^{9,10}$. De acordo com a literatura, os achados dos casos apresentados, corroboram com as características já descritas.

Acredita-se que em ambos os casos descritos, o principal fator desencadeante do trombo vascular tenha sido lesão endotelial. Quando a integridade do revestimento vascular é lesada, expõe-se o tecido 
conjuntivo subepitelial do vaso sanguíneo, permitindo contato direto com o sangue, o que ocasiona adesão e agregação plaquetária e redução dos fatores anticoagulantes. A lesão pode ocorrer por alterações na parede vascular devido a trauma, inflamação, imunopatias, tumores, lesões degenerativas, produtos químicos, drogas e hormônios ${ }^{4}$.

O paciente L.C.M. era portador de prótese parcial removível superior, que, pelo atrito durante fala ou mastigação junto ao dispositivo, pode ter desencadeado a lesão no endotélio. Este paciente relatou ser hipertenso, realizar acompanhamento com cardiologista e tratamento com anti-hipertensivos assiduamente. Alterações no fluxo sanguíneo também podem ter contribuído com o quadro, já que favorecem a agregação de hemácias e plaquetas, lesionando o endotélio dos vasos ${ }^{7}$.

Já o paciente F.M.V. apresentava um possível trauma de mordida na região da lesão. Outro fator que pode desencadear a formação de trombos vasculares é a hipercoagulabilidade congênita ou adquirida. Mutações são as causas congênitas mais frequentes, especialmente, uma alteração genética na molécula do fator $\mathrm{V}$ (interfere na atuação da proteína C que é um dos fatores reguladores do sistema de coagulação). Em condições adquiridas, pode haver aumento da coagulabilidade do sangue por liberação de tromboplastina no plasma, que ativa a via extrínseca da coagulação.

Em estudo que relatava 122 casos de trombose oral ${ }^{1}$, observou-se que $47 \%$ das hipóteses clínicas iniciais foram malformações vasculares, que são associadas à presença de vasos tortuosos indutores de aderência e agregação plaquetária. Uma veia varicosa é a principal causa de trombose em veias superficiais ${ }^{1}$. Os casos descritos acima, podem ser sugestivos de traumas na região como fatores para o surgimento de trombos em mucosa bucal, o que é incomum. $\mathrm{O}$ acompanhamento médico deve ser realizado a fim de evitar que vasos mais profundos e de maior calibre sejam afetados.

\section{CONSIDERAÇÕES FINAIS}

A trombose venosa em boca é incomum e pode ser indicativa de predisposição em outros órgãos. Devido à baixa incidência de trombose em boca, a maioria dos cirurgiões dentistas não leva como principal hipótese diagnóstica. Este profissional tem papel fundamental no reconhecimento das características clínicas e na orientação do paciente quanto à predisposição para formação de trombos. Assim, a anamnese correta associada ao exame clínico e atualização profissional são de extrema relevância para um rápido diagnóstico e imediato tratamento ou encaminhamento ao profissional indicado, pois o diagnóstico histopatológico de trombose exige tal conduta.

\section{REFERÊNCIAS}

1. Tobouti PL, Pigatti FM, Martins-Mussi MC, Machado de Sousa SCO. Oral Thrombus: Report of 122 cases with clinically descriptive data. Med Oral Patol Oral Cir Bucal. 2017;22(3):e366-70.

2. Brandão GMS, Sobreira ML, Rollo HA. Recanalização após trombose venosa profunda aguda. J vasc bras. 2013;12(4):296-302,

3. Kumar DR, Hanlin E, Glurich I, Mazza JJ, Yale SH. Virchow's contribution to the understanding of thrombosis and cellular biology. Clin Med Res. 2010; 8(3-4):168-72.

4. Tjioe KC, Oliveira DT, Santos PS. Tongue phlebothrombosis: Pathogenesis and potential risks. Quintessence Int. 2015;46(6):545-48.

5. Maia M, Cruz A, Vidoedo J, Pinto JA. Síndrome pós-trombótica e qualidade de vida em doentes com trombose venosa ilio. Angiol Cir Vasc. 2014;10(4):173-79.

6. Fernandes DT. Análise clínica de lesões vasculares bucais tratadas cirurgicamente [dissertação]. Piracicaba: Universidade Estadual de Campinas, UNICAMP; 2013.

7. Brasileiro Filho GB. Patologia Geral. 3. ed. Rio de Janeiro: Guanabara Koogan; 2004. p.104-109.

8. Almeida FS, Hossotani MH, Moura JDG. Trombose de artéria carótida interna relacionada a trauma de palato em criança. Rev Paul Pediatr. 2012;30(1):144-47.

9. Corrêa PH, Nunes LC, Johann AC, Aguiar MC, Gomez RS, Mesquita RA. Prevalence of oral hemangioma, vascular malformation and varix in a Brazilian population. Braz Oral Res. 2007; 21(1):40-5.

10.Fonseca Junior NL, Cha SB, Cartum J, Rehder JRCL. Eficácia terapêutica do interferon alfa em criança com hemangioma gigante craniofacial: relato de caso. Arq Bras Oftalmol 2008; 71(3):423-26.

\section{CONFLITO DE INTERESSES}

Os autores declaram não haver conflitos de interesse.

\section{AUTOR PARA CORRESPONDENCIA}

\section{Rosana Mara Giordano de Barros}

rosana-giordano@bol.com.br

Submetido em 07/12/2018

Aceito em 12/03/2019 